\title{
Chronic Kidney Disease in Children: Recent Update
}

\author{
Kwanchai Pirojsakul, Nisha Mathews and Mouin G. Seikaly ${ }^{*}$ \\ Department of Pediatrics, University of Texas Southwestern Medical Center, Children's Medical Center of Dallas, USA
}

\begin{abstract}
The incidence of end stage of renal disease (ESRD) in US children age 0-19 years is 12.9 per million/year (2012). The economic and social burden of diagnosing, treating and preventing chronic kidney disease (CKD) in children and adults remains substantial. Advances in identifying factors that predict development of CKD and its progression, as well as advances in the management of co-morbid conditions including anemia, cardiovascular disease, growth, mineral and bone disorder, and neurocognitive function are discussed. Despite recent reports from retrospective registry data analysis and multi-center prospective studies which have significantly advanced our knowledge of CKD, and despite advances in the understanding of the pathogenesis, diagnosis and treatment of CKD much work remains to be done to improve the long term outcome of this disease.
\end{abstract}

Keywords: Anemia, bone disorder, cardiovascular disease, chronic kidney disease, hypertension, neurocognitive deficit, proteinuria, growt.

\section{INTRODUCTION}

The economic and societal burden of diagnosing, treating and preventing chronic kidney disease (CKD) in children and adults remains substantial. In the US, the incidence of end stage renal disease (ESRD) in children age 0-19 years was 12.9 per million/year in 2012 compared to 352.6 per million/year in all age groups [1]. Efforts are ongoing to improve early diagnosis, intervention and long term outcome. Recent reports from retrospective registry data analysis and multi-center prospective studies significantly advanced as per our knowledge. In this report we will review recent data from North American Pediatric Renal Trials and Cooperative Studies (NAPRTCS), Kidney Disease Outcomes Quality Initiative (KDOQI), Kidney Disease Improving Global Outcomes (KDIGO), Chronic Kidney Disease in Children (CKID) studies, International Society for Peritoneal Dialysis (ISPD), as well as salient individual studies.

\section{SOURCES OF BEST CLINICAL PRACTICE GUIDELINES AND STUDIES}

\subsection{KDOQI (Kidney Disease Outcomes Quality Initiative)}

In 2003, National Kidney Foundation proposed the new definition and staging for CKD in children [2]. Patient with CKD defines as functional or structural damage of the kidney or decrease in glomerular filtration rate (GFR) to less than $60 \mathrm{ml} / \mathrm{min} / 1.73 \mathrm{~m}^{2}$ for more than 3 months. Damage to the kidney can be manifested by abnormalities in blood,

*Address correspondence to this author at the Department of Pediatrics, University of Texas Southwestern Medical Center, Dallas, Texas, TX 75390-9063, USA;

E-mail: mouin.seikaly@utsouthwestern.edu urine, imaging test or pathology on the kidney biopsy. Staging of CKD is classified by GFR as shown in Table $\mathbf{1}$.

Table 1. K/DOQI classification of the stages of chronic kidney disease.

\begin{tabular}{|c|c|}
\hline Stage & GFR $\left(\mathbf{m l} / \mathbf{m i n} / \mathbf{1 . 7 3} \mathbf{m}^{\mathbf{2}}\right)$ \\
\hline \hline 1 & $\geq 90$ \\
\hline 2 & $60-89$ \\
\hline 3 & $30-59$ \\
\hline 4 & $15-29$ \\
\hline 5 & $<15$ \\
\hline
\end{tabular}

\subsection{KDIGO (Kidney Disease - Improving Global Outcomes)}

In 2013, KDIGO published new definition and classification of CKD. CKD is defined as presence of abnormalities of kidney structure or function for more than 3 months. Abnormalities consist of either of albuminuria, urine sediment abnormalities, electrolyte and other abnormalities due to tubular disorders, abnormalities detected by histology, structural abnormalities detected by imaging, or history of kidney transplantation [3]. The KDIGO classification of the stages of chronic kidney disease is shown in Table 2.

\subsection{North American Pediatric Renal Trials and Collaborative Studies (NAPRTCS)}

NAPRTCS was initiated in 1987 aimed to collect retrospective data from pediatric renal transplant and dialysis patients. It has expanded to include pediatric CKD patients since 1992. Recent data from NAPRTCS about anemia and growth in pediatric CKD patients will be discussed [4-6]. 
Table 2. KDIGO classification of the stages of chronic kidney disease.

\begin{tabular}{|c|c|c|}
\hline Stage & GFR $\left(\mathbf{m l} / \mathbf{m i n} / \mathbf{1 . 7 3 \mathbf { m } ^ { 2 } )}\right.$ & Terms \\
\hline \hline 1 & $\geq 90$ & Normal or high \\
\hline 2 & $60-89$ & Mildly decreased \\
\hline $3 \mathrm{a}$ & $45-59$ & Mildly to moderately decreased \\
\hline $3 \mathrm{~b}$ & $30-44$ & Moderately to severed decreased \\
\hline 4 & $15-29$ & Severely decreased \\
\hline 5 & $<15$ & Kidney failure \\
\hline
\end{tabular}

\subsection{Chronic Kidney Disease in Children Study (CKiD Study)}

CKiD study is a prospective observational multi-center study. It was initiated in 2005 by Warady et al. [7] The study has enrolled more than 500 pediatric CKD patients aged 1-16 years old who had eGFR between $30-90 \mathrm{ml} / \mathrm{min} / 1.73 \mathrm{~m}^{2}$. Recent data from this cohort has been published and will be discussed later.

\subsection{International Society for Peritoneal Dialysis (ISPD)}

ISPD is an International society aimed to advance knowledge of peritoneal dialysis and to promote advancement of such knowledge through scientific meetings and publications. In 2012, ISPD developed practice guideline for the management of peritonitis in pediatric patients with peritoneal dialysis [8].

\section{ADVANCES IN REFINING MARKERS OF CKD}

Glomerular filtration rate (GFR) is the best functional marker representing overall renal function. The ideal method for estimation of GFR is inulin clearance [9]. However; measuring inulin clearance requires constant intravenous infusion of inulin and serial urine collection often through bladder catheterization, in young children. Iohexol and Iothalamate are additional markers that have been used to measure GFR. Single intravenous injection of ${ }^{125} \mathrm{I}-$ iothalamate (m-GFR) with repeated blood sampling is used to calculate with plasma disappearance. The CKiD study used iohexol as another marker for GFR. However, these methods are costly and labor intensive. While iothalamte is, Iohexol to date is not FDA approved to measure GFR in the US. Either method is not ideal method for bedside practice. Over the past few years, several formulas were developed to estimate GFR. These formulas were proven to be cohort specific [10]. Practically, the well-known bedside method for GFR estimation is the calculation of the Schwartz's formula as shown below.

eGFR $=\mathrm{k} x$ height $(\mathrm{cm}) /$ serum creatinine $(\mathrm{mg} / \mathrm{dl})$

The value of $\mathrm{k}$ is 0.45 for term infant, 0.55 for children and adolescent girl and 0.7 for adolescent boy [11-13]. These $\mathrm{k}$ values were derived from the study using Jaffe chromogen reaction for measurement of serum creatinine $(\mathrm{sCr})$. Recently, serum creatinine assay has been changed to enzymatic method. The new $\mathrm{k}$ value also has been re- adjusted to 0.413 for patient aged 1-16 year regardless of gender as it has been shown in the CKiD study [14]. Nonetheless, serum creatinine is still not the good marker as its production is related to overall muscle mass of the patients [15]. Thus, estimation of GFR using serum creatinine in CKD patients with malnutrition may be overestimated.

Cystatin $\mathrm{C}$ is another endogenous substance that has been proposed to correlate well with renal function. It is freely filtered by glomerular filtration and completely re-absorbed and metabolized by proximal tubules [9]. It is produced not only by muscle cells but by all nucleated cells. Schwartz et $a l$. has shown that taking serum cystatin $\mathrm{C}$ (sCysC) into the creatinine-based equation as shown below improved GFR estimation with $91 \%$ of eGFR within $\pm 30 \%$ of mGFR [16].

eGFR $=39.8 \times[\text { height }(\mathrm{m}) / \mathrm{sCr}(\mathrm{mg} / \mathrm{dl})]^{0.456} \mathrm{x}$ $[1.8 / \mathrm{sCysC}(\mathrm{mg} / \mathrm{L})]^{0.418}$

$\mathrm{x}[30 / \mathrm{BUN}(\mathrm{mg} / \mathrm{dl})]^{0.079} \mathrm{x} 1.076^{\mathrm{male}} \mathrm{x}[\text { height }(\mathrm{m}) / 1.4]^{0.179}$

Nonetheless, we have shown that this formula is not very accurate in estimation of GFR in the transplant population [10].

\section{ADVANCES IN IDENTIFYING FACTORS THAT PREDICT DEVELOPMENT OF CKD AND ITS PROGRESSION}

\subsection{Hypertension}

Hypertension is one of the modifiable co-morbid variables that should be evaluated and managed properly in children with CKD. An increase in blood pressure (BP) causes increase intra-glomerular pressure and hyper-filtration that leads to progressive deterioration of renal function [17]. The CKiD study has shown that as high as $64 \%$ of pediatric CKD patients required anti-hypertensive medications to control their BP [18]. Furthermore, comparing between the patients with uncontrolled BP and controlled BP, uncontrolled group used significantly less renin-angiotensin antagonists than the controlled group. Some pediatric CKD patients may have normal BP in the clinic but they have elevated BP over the period of 24 hours (masked HT). Samuels et al. [19] conducted a cross sectional study about ambulatory blood pressure monitoring (ABPM) pattern in children with CKD. Interestingly, among 332 CKD patients, $35 \%$ of patients were found to have masked HT diagnosed by ABPM. Moreover, patients who were on ACE-inhibitor also were $89 \%$ more likely to have normal ABPM than those who did not. These patients with masked HT were also found to have left ventricular hypertrophy as much as the patients with confirmed HT [20]. Thus, renin-angiotensin antagonist (ACE-I or ARB) should be considered as the first line regimen in pediatric CKD patients with HT. ABPM also should be used for diagnosis and monitoring of HT in pediatric CKD patients.

The optimal target of BP in pediatric CKD patient has also been studied in the randomized control study "Effect of strict $\mathrm{BP}$ control and $\mathrm{ACE}$ inhibition on progression of CKD in pediatric patients" also known as "ESCAPE trial" [21]. It has shown that intensified BP control showed a substantial benefit on slowing CKD progression in pediatric patients. 
This study enrolled 385 children aged 3-18 years of age with eGFR between $15-80 \mathrm{ml} / \mathrm{min} / 1.73 \mathrm{~m}^{2}$. The patients were randomly assigned to intensified blood pressure control (mean 24 hours BP below $50^{\text {th }}$ percentile) or conventional BP control (mean 24 hours BP between $50^{\text {th }}-95^{\text {th }}$ percentiles). The intervention for baseline BP medication in both groups was ramipril $6 \mathrm{mg} / \mathrm{m}^{2} /$ day. The other $\mathrm{BP}$ medications except renin-angiotensin antagonists were added in order to achieve BP goal in each group. After 5 year follow up, only $29.9 \%$ of the patients in the group of intensified blood-pressure control reached the primary end point (progressed to CKD stage 5 or $50 \%$ decline in GFR) compared with $41.7 \%$ in the group of conventional BP control (hazard ratio, 0.65 with confidence interval, 0.44 to $0.94 ; \mathrm{P}=0.02$ ). This reno-protective effect of lowering $\mathrm{BP}$ is independent of using renin-angiotensin antagonist. These findings have not yet been validated using casual BP readings. This study further suggests that ABPM confers additional benefit to office BP monitoring that could miss silent HT. The effect of other anti-hypertensive medication that targets the renin-angiotensin system such as angiotensin receptor or renin blocker, on progression of CKD awaits further studies.

\subsection{Proteinuria}

Proteinuria has been shown to be associated with progression of CKD in adult population. In the pediatric population, there was a randomized control trial of low protein diet on the progression of CKD [22]. There was no benefit of low protein diet to slow progression of CKD course. In multivariate analysis, proteinuria was one of the predictor of CKD progression. As in the adult study, pediatric patients who had glomerular cause of CKD were found to have lower proteinuria associated with treatment of renin-angiotensin antagonist [23]. Moreover, CKiD investigators recently found that two-fold higher level of baseline proteinuria was associated with faster rate of GFR decline about $0.3 \mathrm{ml} / \mathrm{min}$ per $1.73 \mathrm{~m}^{2}$ per year $(95 \% \mathrm{CI}, 0.4$ to 0.1) in non-glomerular origin of CKD [24]. Thus, treatment with renin-angiotensin antagonist would be essential for pediatric CKD patients with the aim to slow declining of GFR.

Interestingly, Omoloja et al. [25] recently reported effect of secondhand smoke exposure in the CKiD study. Investigators found that patients who reported secondhand smoke exposure had higher urine protein/creatinine ratio compared to the group without smoke exposure $(0.6 \mathrm{vs} 0.4$, $\mathrm{p}<0.01$ ). Smoke exposure in this study was confirmed by higher urinary cotinine level (metabolite of nicotine) in the group with smoke exposure. The pathogenesis of smoke exposure and deterioration of renal function remains unclear. Jaimes et al. [26] discovered that nicotine in the tobacco activates proliferation of mesangial cell and mesangial matrix via nicotinic acetylcholine receptors. Nicotine also increases production of reactive oxygen species and also may lead to inflammation of mesangial matrix [26].

\subsection{Oxidative Stress}

Oxidative stress is an imbalance in the reactive oxygen species (ROS) production/degradation ratio. Under normal conditions, ROS (which include various compounds such as superoxide anions, hydrogen peroxide, and hydroxylradical) can accelerate renal injury progression. Inflammatory markers such as C-reactive protein and cytokines increase with renal function deterioration suggesting that CKD is a low-grade inflammatory process. In fact, inflammation facilitates renal function deterioration. Several factors can be involved in triggering the inflammatory process including oxidative stress. Statin administration is accompanied by risk reduction in all major vascular events in high risk CKD patients. These beneficial effects seem to be a consequence of their hypolipidemic as well as their direct effect [27].

\subsection{Low Birth Weight, Prematurity and Risk for Progression of CKD}

The metanephros gives rise to definitive kidneys which continue to develop until 36 weeks of gestation. Although glomerulogenesis still occurs in premature infant, many of the glomeruli are abnormal such as cystic dilatation [28]. Another study by Mañalich et al. [29] showed that low nephron number was directly correlated with birth weight and inversely correlated with glomerular size. These infants have low nephron number and abnormal glomeruli leading to hyper-filtration of the remaining glomeruli, proteinuria and CKD later in life [30].

\section{Advances in the Management of Co-morbid Conditions}

\subsection{Anemia}

Anemia is a common co-morbid condition in pediatric CKD. Report from NAPRTCS in 2010 has found that as high as $73 \%$ of children with CKD stage $3,87 \%$ of CKD stage 4 , and over $93 \%$ of CKD stage 5 were anemic. (4) Anemia has been shown to be associated with risk of hospital admission. Staples et al. [31] investigated 2,779 pediatric CKD patients from NAPRTCS and found that patients with anemia (hematocrit $<33 \%$ ) were $55 \%$ more likely to be hospitalized that non-anemic patients. The causes of anemia in pediatric CKD patients include iron deficiency, blood loss, erythropoietin deficiency and anemia of chronic disease. These factors have varying effects at the particular stage of CKD. Iron deficiency is more prominent cause at the early stage of CKD but erythropoietin deficiency is major cause at the late stage. Baracco et al. [32] investigated 50 pediatric CKD patients and found that $25 \%$ of patients with stage 2 and $55.5 \%$ of patients with CKD stage 3 had iron deficiency defined by low transferrin saturation $(<20 \%)$ and low serum ferritin for age. Thus, pediatric CKD patients with anemia should be worked up for iron deficiency before starting erythropoietin stimulating agent (ESA).

In 2013, KDIGO published the new recommendation about target hemoglobin in pediatric CKD patients. Anemia is defined as hemoglobin concentration of $<11.0 \mathrm{~g} / \mathrm{dl}$ in children $0.5-5$ years, $<11.5 \mathrm{~g} / \mathrm{dl}$ in children $5-12$ years, and $<12.0 \mathrm{~g} / \mathrm{d}$ l in children $12-15$ years [3]. Generally, pediatric CKD patients require larger starting dose of ESA (200-300 units/kg/week) comparing to adult CKD patients (100-150 units $/ \mathrm{kg} /$ week) [31]. Over treatment of ESA with higher level of hemoglobin than $13 \mathrm{~g} / \mathrm{dl}$ is not recommended as it 
has been shown to increase cardiovascular events in the adult population. However, no pediatric data has been reported [33]. Patients who require higher dose of ESA should be investigated for blood loss, iron deficiency, secondary hyperparathyroidism, inflammation from original disease etc. Vitamin D deficiency also needs to be in the differential diagnosis as it has been shown that treatment of vitamin D deficiency decreased the dosages of ESA in pediatric CKD patients [34,35].

Hepcidin, an acute phase protein made from liver, has been recently discovered and found to be elevated in pediatric CKD patients. High hepcidin level inhibits iron absorption from the intestine and impairs release from body storage through the inhibition of ferroportin [36]. Meredith et al. [37] has reported from CKiD cohort that higher hepcidin level is associated with decreased hemoglobin especially in those with low GFR. Although, serum hepcidin has been found to decrease with hemodialysis or treatment with erythropoietin stimulating agent (ESA), treatment effect of lowering hepcidin on improvement of anemia in pediatric patients has never been reported [38,39]. Nonetheless, hepcidin is currently the prospective target for the treatment of anemia in CKD patients as it has been shown in the animal model that the LDN-193189 (hepcidin lowering agent) lowered hepatic hepcidin mRNA and mobilized iron storage into plasma and increased hemoglobin content of reticulocytes in the adenine-induced CKD rats [40].

Replenishing iron stores to a transferrin saturation of 20$30 \%$ is desirable. The objective of iron management is to maintain Hemoglobin in the 11-12 g/dl range. Due to relative Fe malabsorption in CKD, oral iron may not be sufficient to achieve this goal and one may have to resort to parentral iron supplementation. Recently, iron formulations (Ferric Citrate and Sucroferric Oxyhydroxide) with the dual purpose of replenishing iron stores and phosphate binding became available. Original studies showed that ferric citrate is superior in delivering iron but patients could run the risk of increased aluminum absorption and toxicity due to the citrate [41].

\subsection{Cardiovascular Disease}

Children with CKD are at an increased risk to develop cardiovascular disease [42]. Parekh et al. [43] has shown that pediatric patient with CKD who were on dialysis had cardiac death rate as high as 20 per 1,000 patient-year. Increase in sympathetic activity has been associated with increase in cardiovascular risk as shown in the adult patient with CKD [44]. The nature of cardiovascular death in children is different than that in adults. In adults, coronary artery disease and congestive heart failure are the leading causes, while in children, cardiomyopathy and arrhythmia are most prevalent [45]. Recently, the CKiD study found that pediatric CKD patients with HT also had decreased heart rate variability and increased BP variability. Both findings are signs of sympathetic nervous system over-activity [46]. Dyslipidemia is also a known risk factor of cardiovascular disease. Saland et al. [47] has shown that $45 \%$ of pediatric CKD patients had dyslipidemia. Low GFR, overweight and nephrotic proteinuria were significantly associated with dyslipidemia. Carotid intima-media (cIMT) thickness is another predictor of cardiovascular events. Brady et al. [48] has found that there is greater cIMT in pediatric patients with CKD than with healthy controls. In multivariate analysis, dyslipidemia and HT were significantly associated with greater cIMT [48]. Thus, CKiD investigators recommend screening pediatric patients with CKD for dyslipidemia especially those who have high BMI, lower GFR and nephrotic proteinuria [49].

\subsection{Growth}

Poor linear growth is a well described and discernable complication of CKD. The etiology of poor growth is multifactorial. Growth hormone-pituitary axis is often maintained. The main issue in later stages of CKD is the low bioavailability of insulin-like growth factor 1 (IGF-1) due to decreased insulin-like growth factor binding protein 3 (IGFBP3) and increase in other carrier proteins. Other factors contributing to poor growth in children with CKD include protein energy wasting, metabolic acidosis, malnutrition, renal osteodystrophy, and medications such as steroids $[49,50]$. It should be emphasized that in CKD stage 3 through CKD stage 5, poor linear growth occurs because of growth hormone resistance rather than deficiency. Some proposed mechanisms of growth hormone resistance include reduced density of growth hormone receptors in target organs, impaired growth hormone-activated post-receptor Janus kinase/signal transducer and activator of transcription signaling, and reduced levels of free IGF-1 due to increased inhibitory IGF-binding proteins [50].

In 2006, Seikaly et al. [51] evaluated the NAPRTCS enrollment registry data of 5,615 children aged birth to 21 years to correlate factors associated with short stature in children with CKD. They found that older patients $(>12$ years), those with GFR $>50 \mathrm{ml} / \mathrm{min} / 1.73 \mathrm{~m}^{2}$, black patients and patients with focal segmental glomerulosclerosis were at lower risk of being short at entry. Anemia (hematocrit below $33 \%$ ) was an independent risk factor for short stature. Acidosis, serum phosphorous, calcium, albumin and PTH at registration were poor predictors of short stature.

Greenbaum et al. [52] identified low birth weight $(<2,500$ grams) and small for gestation age (birth weight $<10$ th percentile for gestational age) as novel risk factors for short stature and lower weight percentiles in children with mild to moderate CKD independent of kidney function from the CKiD cohort. In another study from the CKiD cohort, Rodig et al. [53] found that girls with non-glomerular CKD were the shortest and compared to those with a serum bicarbonate $\left(\mathrm{HCO}_{3}\right)$ level of $\geq 22 \mathrm{mEq} / \mathrm{L}$, children with $\mathrm{HCO}_{3}$ of $<18 \mathrm{mEq} / \mathrm{L}$ had a height standard deviation score (SDS) that was on average 0.67 lower. Only $23 \%$ of children with a height SDS of $\leq-1.88$ were prescribed growth hormone therapy [53]. Pharmacological doses of recombinant human growth hormone $(\mathrm{rhGH})$ can improve linear growth. Children should have hip x-rays and a wrist bone age prior to initiation of therapy. From the NAPRTCS registry, it was shown that long term rhGH therapy enhances height velocity for at least 2.5 years, was well tolerated without an increase in the rate of progression of CKD, and had no effect on BMI [5]. Mahan et al. [54] constructed growth curves for assessing a patient's first year of growth response from Genentech's National Cooperative Growth Study and proposed that a height velocity below the mean $1 \mathrm{SD}$ was an inadequate response. 
KDOQI recommends a dose of $\mathrm{rhGH}$ of $0.05 \mathrm{mg} / \mathrm{kg} / \mathrm{day}$ or $30 \mathrm{IU} / \mathrm{m}^{2} /$ week as a subcutaneous injection with close monitoring of calcium, phosphorus, $\mathrm{PTH}$, and alkaline phosphatase. In a recent study by Akchurin et al. [55] examining medication adherence and growth; it was found that self-reported non adherence to rhGH was associated with poorer growth velocity in children with CKD.

\subsection{CKD - Mineral and Bone Disorder}

CKD causes disordered regulation of mineral metabolism with subsequent alterations in skeletal and cardiovascular biology which is now referred to as CKD - mineral and bone disorder (CKD-MBD) [56]. Hyperparathyroidism in advanced CKD is secondary to the deficiency of 1,25 dihydroxyvitamin $\quad \mathrm{D} \quad\left(1,25 \quad(\mathrm{OH})_{2} \mathrm{D}\right)$ combined with hyperphosphatemia leading to abnormal bone turnover and mineralization. Fibroblast growth factor 23 (FGF23) is a bone derived circulating hormone that inhibits renal phosphate reabsorption and suppresses the synthesis of 1,25 $(\mathrm{OH})_{2} \mathrm{D}$ thereby acting as a phosphaturic hormone. Circulating FGF23 was significantly elevated in patients with CKD and its concentration correlated with renal creatinine clearance [57]. FGF23 normalizes serum phosphate and decreases $1,25(\mathrm{OH})_{2} \mathrm{D}$ levels in early stage $\mathrm{CKD}$, and suggests a pathological sequence of events for the development of secondary hyperparathyroidism triggered by increased FGF23, followed by a reduction of $1,25(\mathrm{OH})_{2} \mathrm{D}$ and calcium levels, thereby increasing parathyroid hormone secretion [58]. Portale et al. [59] found that serum FGF23 is the earliest detectable abnormality in mineral metabolism, and levels are highest in glomerular diseases. Serum phosphorus levels, adjusted for age, were significantly lower at GFR of $60-69 \mathrm{ml} / \mathrm{min}$ per $1.73 \mathrm{~m}^{2}$ than higher GFR, but thereafter they became higher in parallel with fibroblast growth factor 23 as GFR declined [59].

Recent studies have shown that induction of vascular calcification begins in early normophosphatemic CKD by the reduction of vascular Klotho and increased FGF23 secretion [60]. Studies of the vasculature in CKD indicate the presence of osteoblastic differentiation in the vessel wall suggesting that uremic serum and high phosphate stimulate osteoblastic differentiation of calcifying vascular cells and vascular smooth muscle cells [61]. Vascular calcifications occurring in the uremic milieu develop primarily in the tunica media contrary to the calcifications of athleroslerotic plaques that develop with age in the vascular intima [62] Data from Faul et al. [63] demonstrated that chronically elevated FGF23 levels have a direct effect on the pathogenesis of left ventricular hypertrophy through a Klotho independent mechanism. There is ongoing research whether FGF-23 is a modifiable risk factor that can be translated into an earlier clinical management of disordered mineral metabolism in CKD [64].

\subsection{Neurocognitive Aspect}

The vulnerability of the central nervous system to atrophy, delayed conduction velocity on electrophysiology studies, and specific cognitive deficits has been identified in CKD patients [65]. A report of baseline neurocognitive function in the CKiD cohort revealed $21 \%$ to $40 \%$ of participants scored at least one standard deviation below the normative data on measures of intelligence quotient, academic achievement, attention regulation, and executive functioning [66]. Higher iohexol-based GFR predicted a lesser risk for poor performance on measures of executive function [66]. Participants having elevated proteinuria (urine protein/creatinine $>2$ ) scored lower on verbal IQ, full-scale IQ, and attention variability than those without elevated proteinuria [66].

Children with CKD perform less well on standardized tests of intelligence and academic achievement than their unaffected siblings [67]. Mendley et al. compared test performance across the range of estimated GFR and duration of CKD with relevant covariates including maternal education, household income, IQ, BP, and preterm birth. It was found that in a population with mild-to-moderate CKD, the duration of disease rather than estimated GFR was associated with impaired attention regulation and inhibitory control [68].

With ongoing pharmacological advances, children with CKD are surviving into adulthood. There has been an increase in the development and utilization of pediatric health-related QOL (HRQOL) measures as a tool to evaluate healthcare services and as an effort to improve patient health and well-being [69]. In the first study to assess depression in pediatric CKD patients living in the USA and the largest study to identify the clinical and demographic factors associated with depression in this patient population, Kogon et al. [70] found a high proportion of children with CKD are depressed and that those with a diagnosis of kidney disease for $>3$ years and those with CKD stage 3 may be particularly susceptible.

\section{CONCLUSION}

Despite recent advances in the understanding of the pathogenesis of CKD and its management, all these findings and recent advancement remain in working progress as much more needs to be elucidated.

\section{ABBREVIATIONS}

$$
\begin{array}{ll}
\mathrm{ABPM} & =\text { Ambulatory blood pressure monitoring } \\
\mathrm{BP} & =\text { Blood pressure } \\
\mathrm{CKD} & =\text { Chronic kidney disease } \\
\mathrm{CKiD} & =\text { Chronic Kidney Disease in Children } \\
\mathrm{ESA} & =\text { Erythropoietin stimulating agent } \\
\mathrm{ESRD} & =\text { End stage renal disease } \\
\mathrm{GFR} & =\text { Glomerular filtration rate } \\
\mathrm{HT} & =\text { Hypertension } \\
\mathrm{ISPD} & =\text { International Society for Peritoneal Dialysis } \\
\mathrm{KDIGO} & =\text { Kidney Disease Improving Global Outcomes } \\
\mathrm{KDOQI} & =\text { Kidney Disease Outcomes Quality Initiative } \\
\text { NAPRTCS } & =\text { North American Pediatric Renal Trials and } \\
&
\end{array}
$$




\section{CONFLICT OF INTEREST}

The authors confirm that this article content has no conflict of interest.

\section{ACKNOWLEDGEMENTS}

Declared none.

\section{REFERENCES}

[1] United States Renal Data System, 2014 Annual Data Report: Epidemiology of Kidney Disease in the United States. [database on the Internet]. 2014 [cited 12/15/2014]. Available from: http://www.usrds.org/2014/view/Default.aspx.

[2] Hogg RJ, Furth S, Lemley KV, et al. National Kidney Foundation's Kidney Disease Outcomes Quality Initiative clinical practice guidelines for chronic kidney disease in children and adolescents: evaluation, classification, and stratification. Pediatrics 2003; 111: 1416-21.

[3] Kidney Disease: Improving Global Outcomes (KDIGO) CKD Work Group. KDIGO clinical practice guideline for the evaluation and management of chronic kidney disease. Kidney Inter Suppl 2013; 3: 1-150.

[4] Atkinson MA, Martz K, Warady BA, et al. Risk for anemia in pediatric chronic kidney disease patients: a report of NAPRTCS. Pediatr Nephrol 2010; 25: 1699-706.

[5] Seikaly MG, Waber P, Warady BA, et al. The effect of rhGH on height velocity and BMI in children with CKD: a report of the NAPRTCS registry. Pediatr Nephrol 2009; 24: 1711-7.

[6] Seikaly MG, Salhab N, Warady BA, et al. Use of rhGH in children with chronic kidney disease: lessons from NAPRTCS. Pediatr Nephrol 2007; 22: 1195-204.

[7] Furth SL, Cole SR, Moxey-Mims M, et al. Design and methods of the Chronic Kidney Disease in Children (CKiD) prospective cohort study. Clin J Am Soc Nephrol 2006; 1:1006-15.

[8] Warady BA, Bakkaloglu S, Newland J, et al. Consensus guidelines for the prevention and treatment of catheter-related infections and peritonitis in pediatric patients receiving peritoneal dialysis: 2012 update. Perit Dial Int 2012; 32(Suppl 2): S32-86.

[9] Schwartz GJ, Furth SL. Glomerular filtration rate measurement and estimation in chronic kidney disease. Pediatr Nephrol 2007; 22: $1839-48$

[10] Siddique K, Leonard D, Borders L, et al. Validation of the CKiD formulae to estimate GFR in children post renal transplant. Pediatr Nephrol 2014; 29: 445-51

[11] Schwartz GJ, Haycock GB, Edelmann CM, et al. A simple estimate of glomerular filtration rate in children derived from body length and plasma creatinine. Pediatrics 1976; 58: 259-63.

[12] Schwartz GJ, Feld LG, Langford DJ. A simple estimate of glomerular filtration rate in full-term infants during the first year of life. J Pediatr 1984; 104: 849-54.

[13] Schwartz GJ, Gauthier B. A simple estimate of glomerular filtration rate in adolescent boys. J Pediatr 1985; 106: 522-6.

[14] Schwartz GJ, Munoz A, Schneider MF, et al. New equations to estimate GFR in children with CKD. J Am Soc Nephrol 2009; 20: 629-37.

[15] Schwartz GJ, Work DF. Measurement and estimation of GFR in children and adolescents. Clin J Am Soc Nephrol 2009; 4: 1832-43.

[16] Schwartz GJ, Schneider MF, Maier PS, et al. Improved equations estimating GFR in children with chronic kidney disease using an immunonephelometric determination of cystatin C. Kidney Int 2012; 82: 445-53.

[17] Schaefer B, Wuhl E. Educational paper: Progression in chronic kidney disease and prevention strategies. Eur J Pediatr 2012; 171: 1579-88.

[18] Flynn JT, Mitsnefes M, Pierce C, et al. Blood pressure in children with chronic kidney disease: a report from the Chronic Kidney Disease in Children study. Hypertension 2008; 52: 631-7.

[19] Samuels J, Ng D, Flynn JT, et al. Ambulatory blood pressure patterns in children with chronic kidney disease. Hypertension 2012; 60: 43-50
[20] Mitsnefes M, Flynn J, Cohn S, et al. Masked hypertension associates with left ventricular hypertrophy in children with CKD. J Am Soc Nephrol 2010; 21: 137-44.

[21] Wuhl E, Trivelli A, Picca S, et al. Strict blood-pressure control and progression of renal failure in children. N Engl J Med 2009; 361: $1639-50$

[22] Wingen AM, Fabian-Bach C, Schaefer F, et al. Randomised multicentre study of a low-protein diet on the progression of chronic renal failure in children. European Study Group of Nutritional Treatment of Chronic Renal Failure in Childhood. Lancet 1997; 349: 1117-23.

[23] Wong CS, Pierce CB, Cole SR, et al. Association of proteinuria with race, cause of chronic kidney disease, and glomerular filtration rate in the chronic kidney disease in children study. Clin J Am Soc Nephrol 2009; 4: 812-9.

[24] Fathallah-Shaykh SA, Flynn JT, Pierce CB, et al. Progression of Pediatric CKD of Nonglomerular Origin in the CKiD Cohort. Clin JASN 2015 [Epub ahead of print].

[25] Omoloja A, Jerry-Fluker J, Ng DK, et al. Secondhand smoke exposure is associated with proteinuria in children with chronic kidney disease. Pediatr Nephrol 2013; 28: 1243-51.

[26] Jaimes EA, Tian RX, Raij L. Nicotine: the link between cigarette smoking and the progression of renal injury? Am J Physiol Heart Circ Physiol 2007; 292: 76-82.

[27] Cachofeiro V, Goicochea M, de Vinuesa SG, et al. Oxidative stress and inflammation, a link between chronic kidney disease and cardiovascular disease. Kidney Int Suppl 2008: S4-9.

[28] Abitbol CL, Rodriguez MM. The long-term renal and cardiovascular consequences of prematurity. Nat Rev Nephrol 2012; 8: 265-74.

[29] Manalich R, Reyes L, Herrera M, et al. Relationship between weight at birth and the number and size of renal glomeruli in humans: a histomorphometric study. Kidney Int 2000; 58: 770-3.

[30] Carmody JB, Charlton JR. Short-term gestation, long-term risk: prematurity and chronic kidney disease. Pediatrics 2013; 131: 1168-79.

[31] Atkinson MA, Furth SL. Anemia in children with chronic kidney disease. Nat Rev Nephrol 2011; 7: 635-41.

[32] Baracco R, Saadeh S, Valentini R, et al. Iron deficiency in children with early chronic kidney disease. Pediatr Nephrol 2011; 26: 2077 80.

[33] KDOQI. KDOQI Clinical Practice Guideline and Clinical Practice Recommendations for anemia in chronic kidney disease: 2007 update of hemoglobin target. Am J Kidney Dis 2007; 50: 471-530.

[34] Bamgbola OF. Pattern of resistance to erythropoietin-stimulating agents in chronic kidney disease. Kidney Int 2011; 80: 464-74.

[35] Rianthavorn P, Boonyapapong P. Ergocalciferol decreases erythropoietin resistance in children with chronic kidney disease stage 5. Pediatr Nephrol 2013; 28: 1261-6.

[36] Atkinson MA, White CT. Hepcidin in anemia of chronic kidney disease: review for the pediatric nephrologist. Pediatr Nephrol 2012; 27: 33-40

[37] Atkinson MA, Kim JY, Roy CN, et al. Hepcidin and risk of anemia in CKD: a cross-sectional and longitudinal analysis in the CKiD cohort. Pediatr Nephrol 2015; 30(4): 635-43.

[38] Ashby DR, Gale DP, Busbridge M, et al. Plasma hepcidin levels are elevated but responsive to erythropoietin therapy in renal disease. Kidney Int 2009; 75: 976-81.

[39] Zaritsky J, Young B, Gales B, et al. Reduction of serum hepcidin by hemodialysis in pediatric and adult patients. Clin J Am Soc Nephrol 2010; 5: 1010-4.

[40] Sun CC, Vaja V, Chen S, et al. A hepcidin lowering agent mobilizes iron for incorporation into red blood cells in an adenineinduced kidney disease model of anemia in rats. Nephrol Dial Transplant 2013; 28: 1733-43.

[41] Negri AL, Ureña Torres PA. Iron-based phosphate binders: do they offer advantages over currently available phosphate binders? Clin Kidney J 2015; 8(2): 161-7.

[42] Muscheites J, Meyer AA, Drueckler E, et al. Assessment of the cardiovascular system in pediatric chronic kidney disease: a pilot study. Pediatr Nephrol 2008; 23: 2233-9.

[43] Parekh RS, Carroll CE, Wolfe RA, et al. Cardiovascular mortality in children and young adults with end-stage kidney disease. J Pediatr 2002; 141: 191-7. 
[44] Converse RL, Toto RD, Jost CM, et al. Sympathetic overactivity in patients with chronic renal failure. N Engl J Med 1992; 327: 19128.

[45] Mitsnefes MM. Cardiovascular complications of pediatric chronic kidney disease. Pediatr Nephrol 2008; 23: 27-39.

[46] Barletta GM, Flynn J, Mitsnefes M, et al. Heart rate and blood pressure variability in children with chronic kidney disease: a report from the CKiD study. Pediatr Nephrol 2014; 29:1059-65.

[47] Saland JM, Pierce CB, Mitsnefes MM, et al. Dyslipidemia in children with chronic kidney disease. Kidney Int 2010; 78: 115463.

[48] Brady TM, Schneider MF, Flynn JT, et al. Carotid intima-media thickness in children with CKD: results from the CKiD study. Clin J Am Soc Nephrol 2012; 7: 1930-7.

[49] Wong CJ, Moxey-Mims M, Jerry-Fluker J, et al. CKiD (CKD in children) prospective cohort study: a review of current findings. Am J Kidnry Dis 2012; 60: 1002-11.

[50] Mahesh S, Kaskel F. Growth hormone axis in chronic kidney disease. Pediatr Nephrol 2008; 23: 41-8.

[51] Seikaly MG, Salhab N, Gipson D, et al. Stature in children with chronic kidney disease: analysis of NAPRTCS database. Pediatr Nephrol 2006; 21: 793-9.

[52] Greenbaum LA, Munoz A, Schneider MF, et al. The association between abnormal birth history and growth in children with CKD. Clin JASN 2011; 6: 14-21.

[53] Rodig NM, McDermott KC, Schneider MF, et al. Growth in children with chronic kidney disease: a report from the Chronic Kidney Disease in Children Study. Pediatr Nephrol 2014; 29: 198795.

[54] Mahan JD, Warady BA, Frane J, et al. First- year response to rhGH therapy in children with CKD: a National Cooperative Growth Study Report. Pediatr Nephrol 2010; 25: 1125-30.

[55] Akchurin OM, Schneider MF, Mulqueen L, et al. Medication adherence and growth in children with CKD. Clin J Am Soc Nephrol 2014; 9: 1519-25.

[56] Kidney Disease: Improving Global Outcomes CKDMBDWG. KDIGO clinical practice guideline for the diagnosis, evaluation, prevention, and treatment of Chronic Kidney Disease-Mineral and Bone Disorder (CKD-MBD). Kidney Int Suppl 2009: S1-130.

[57] Larsson $\mathrm{T}$, Nisbeth $\mathrm{U}$, Ljunggren $\mathrm{O}$, et al. Circulating concentration of FGF-23 increases as renal function declines in patients with chronic kidney disease, but does not change in response to variation in phosphate intake in healthy volunteers. Kidney Int 2003; 64: 2272-9.

[58] Hasegawa H, Nagano N, Urakawa I, et al. Direct evidence for a causative role of FGF23 in the abnormal renal phosphate handling and vitamin D metabolism in rats with early-stage chronic kidney disease. Kidney Int 2010; 78: 975-80.

[59] Portale AA, Wolf M, Juppner H, et al. Disordered FGF23 and mineral metabolism in children with CKD. Clin J Am Soc Nephrol 2014; 9: 344-53.

[60] Paoli S, Mitsnefes MM. Coronary artery calcification and cardiovascular disease in children with chronic kidney disease. Curr Opin Pediatr 2014; 26: 193-7.

[61] Hruska KA, Choi ET, Memon I, et al. Cardiovascular risk in chronic kidney disease (CKD): the CKD-mineral bone disorder (CKD-MBD). Pediatr Nephrol 2010; 25: 769-78.

[62] Moe SM, Chen NX. Mechanisms of vascular calcification in chronic kidney disease. J Am Soc Nephrol 2008; 19: 213-6.

[63] Faul C, Amaral AP, Oskouei B, et al. FGF23 induces left ventricular hypertrophy. J Clin Invest 2011; 121: 4393-408.

[64] Larsson TE. The role of FGF-23 in CKD-MBD and cardiovascular disease: friend or foe? Nephrol Dial Transplant 2010; 25: 1376-81.

[65] Gipson DS, Duquette PJ, Icard PF, European Renal Association. The central nervous system in childhood chronic kidney disease. Pediatr Nephrol 2007; 22: 1703-10.

[66] Hooper SR, Gerson AC, Butler RW, et al. Neurocognitive functioning of children and adolescents with mild-to-moderate chronic kidney disease. Clin J Am Soc Nephrol 2011; 6:1824-30.

[67] Brouhard BH, Donaldson LA, Lawry KW, et al. Cognitive functioning in children on dialysis and post-transplantation. Pediatr Transplant 2000; 4: 261-7.

[68] Mendley SR, Matheson MB, Shinnar S, et al. Duration of chronic kidney disease reduces attention and executive function in pediatric patients. Kidney Int 2015; 87(4): 800-6.

[69] Matza LS, Swensen AR, Flood EM, et al. Assessment of healthrelated quality of life in children: a review of conceptual, methodological, and regulatory issues. Value Health 2004; 7: 7992.

[70] Kogon AJ, Vander Stoep A, Weiss NS, et al. Depression and its associated factors in pediatric chronic kidney disease. Pediatr Nephrol 2013; 28:1855-61. 